Cahiers de recherches médiévales

Journal of medieval studies

$8 \mid 2001$

La protection spirituelle au Moyen Âge

\title{
L'idée de croisade dans deux œuvres de Christine de Pizan
}

Claire Le Ninan

\section{(2) OpenEdition \\ 12 Journals}

Édition électronique

URL : https://journals.openedition.org/crm/411

DOI : $10.4000 / \mathrm{crm} .411$

ISSN : 1955-2424

Éditeur

Honoré Champion

\section{Édition imprimée}

Date de publication : 15 janvier 2001

Pagination : 251-261

ISSN : 1272-9752

\section{Référence électronique}

Claire Le Ninan, "L'idée de croisade dans deux œuvres de Christine de Pizan », Cahiers de recherches médiévales [En ligne], 8 | 2001, mis en ligne le 30 novembre 2009, consulté le 15 décembre 2022. URL: http://journals.openedition.org/crm/411 ; DOl : https://doi.org/10.4000/crm.411

Ce document a été généré automatiquement le 15 décembre 2022.

Tous droits réservés 


\title{
L'idée de croisade dans deux œuvres de Christine de Pizan
}

\author{
Claire Le Ninan
}

1 Alors que certains auteurs de la fin du XIV siècle, tels Eustache Deschamps ${ }^{1}$ et surtout Philippe de Mézières ${ }^{2}$, encouragent les princes français à combattre les musulmans et à reconquérir la Terre Sainte, Christine de Pizan appelle plus modérément à lutter contre les «Sarrasins». Comme le remarque Berenice A.Carroll dans un article récent ${ }^{3}$, Christine n'aborde pas le débat sur la légitimité de la guerre contre les musulmans dans le Livre des fais d'armes et de chevalerie, bien que cette question soit discutée dans la source principale de ce traité, L'Arbre des batailles d'Honoré Bouvet. En revanche, la dernière œuvre de l'écrivain, le Ditié de Jehanne d'Arc ${ }^{4}$, défend vivement l'idée de croisade. Berenice A. Caroll, en s'appuyant sur un article d'Earl Jeffrey Richards ${ }^{5}$, explique cette différence de point de vue par le fait qu'au début de sa carrière, le nationalisme de Christine ne présente pas les accents de chauvinisme qu'il aura à la fin ${ }^{6}$. Pourtant, si on examine les premières œuvres politiques de l'écrivain, on constate que l'idée de croisade, bien que discrète, n'en est pas absente. Plus précisément, ses manifestations semblent dépendre de la forme littéraire du texte qui traite des musulmans. Le Livre du chemin de long estude $e^{7}$ et le Livre des fais et bonnes meurs du sage roy Charles $V^{8}$, écrits à deux ans d'intervalle, 1402 et 1404, et dont l'objet commun est de proposer un modèle de roi idéal dans lequel la sagesse tient une place prépondérante, contiennent l'un et l'autre quelques-uns des rares passages de son œuvre où Christine s'intéresse à l'Orient et aux musulmans. Dans le premier de ces textes, la réflexion sur les conflits du monde précédant l'endormissement et le voyage dans les différentes régions de la Terre à la suite de la Sibylle fournissent à l'auteur l'occasion de mentionner les «Sarrasins ", terme qui désigne les musulmans en général, qu'ils soient Ottomans, Mongols ou Mamelouks. Dans le Charles V, un long passage du chapitre XXXI du livre III raconte la visite d'un ambassadeur du sultan d'Égypte à la cour de France.

2 L'écrivain adopte cependant d'un texte à l'autre des stratégies stylistiques et narratives très différentes. Le Chemin de long estude prend la forme d'un songe allégorique, écrit en vers. Christiane Marchello-Nizia ${ }^{9}$ a montré que le cadre du rêve inscrit le texte dans la 
fiction. Ces éléments font du Chemin de long estude une œuvre littéraire, qui met une distance entre les vérités qu'elle énonce et le lecteur grâce à la construction poétique. Le Charles $V$, au contraire, par l'écriture en prose et par l'affirmation dès le début de l'œuvre de son caractère historique ${ }^{10}$, se veut une description fidèle de la vie du roi et de ses vertus. Or à cette différence de nature littéraire s'ajoute une distinction dans la façon de percevoir les musulmans. Si l'idée de croisade est présente dans le Chemin de long estude, elle semble en premier lieu absente du Charles $V$.

3 Dans le Chemin de long estude, la déploration des conflits du monde qui précède l'endormissement semble orienter l'ensemble du songe qui suit vers la recherche de la paix pour l'humanité entière. Si l'équilibre de Nature justifie la lutte d'éléments et d'animaux qui sont d'espèces différentes, ce n'est pas le cas des hommes qui appartiennent à la même espèce et donc devraient vivre en paix ${ }^{11}$. Pourtant, l'étude des passages concernant les «Sarrasins » laisse percevoir une hostilité à leur égard qui va à l'encontre du projet initial de l'œuvre. Que ce soit dans la méditation sur les conflits, dans la contemplation des ruines de Constantinople ou dans le récit de la visite du Proche-Orient, Christine ne se contente pas de signaler la présence des musulmans ou l'existence de luttes qui les déchirent, elle exprime également une opinion très négative sur ces derniers :

Et meisme entre les sarrasins,

Le basat contre Tambourlan -

Que Dieux mette en si tres mal an

Qu'ilz se puissent entre eulx deffaire,

Si n'y ait crestiens que faire ! (vv. 346-350)

Mais trop plaignoie les dommages

Des ruines de ceste ville,

Ou il y a en plus de mille

Lieux les haulx murs tous cheus par terre

Par meschef et par longue guerre

Qu'ilz ont tout temps aux Sarrasins,

Qui trop leur sont prochains voisins. (vv. 1220-1226)

Voult celle que par la alasse

Veoir la terre du Souldan

Qui aux Crestiens fait maint dan. (vv. 1316-1318)

Si vi tout la region

Et la court de cel empereur

Qui tant est grant que c'et orreur,

Tout ait il guerre au Tamburlan

Qui le destruira, ce dit l'en. (vv. 1326-1330)

Les «Sarrasins » sont représentés comme une puissance effrayante et dangereuse, en lutte contre les chrétiens. Cette impression est renforcée, dans les deux dernières citations $^{12}$, par l'association à la rime de termes désignant le monarque avec d'autres soulignant son action néfaste : « souldan » se trouve ainsi lié à « dan » et « empereur » à " orreur».

Les vers exprimant un jugement péjoratif n'ont pas de réelle utilité dans l'économie du récit. En introduisant dans la narration des éléments extérieurs au monde fictif du songe, Christine brise aussi l'unité temporelle du récit. En effet, le voyage onirique bénéficie d'une sorte d'atemporalité : il est ponctué uniquement par la succession des lieux, en dehors de toute mesure précise du temps. En émettant un avis sur les musulmans et surtout en citant le nom du chef mongol, Tamerlan, Christine rompt avec l'atemporalité et fait émerger la réalité historique dans la fiction. La menace sarrasine 
sur Constantinople et la guerre de Tamerlan contre le sultan de Babylone évoquent des événements contemporains, en rapport avec la date mentionnée avant l'endormissement ${ }^{13}$.

6 Il faut sans doute voir dans les critiques de Christine envers les musulmans une résurgence des traumatismes laissés dans les esprits occidentaux par les échecs des différentes tentatives de croisade de Charles VI: la croisade de Barbarie en 1390 et surtout la bataille de Nicopolis en 1396 qui a vu la défaite des nobles français et anglais, unis pour défendre le roi de Hongrie contre Bayazid ${ }^{14}$. Pourtant, le présent employé dans les trois dernières citations fait référence à des situations contemporaines, ce qui laisse envisager une explication liée à l'actualité immédiate. Ainsi nous pouvons sans doute percevoir dans cette insistance sur le caractère menaçant des "Sarrasins » une tentative pour inciter le roi de France à se battre de nouveau contre ces ennemis qui offensent la foi chrétienne. Plusieurs éléments textuels nous permettent d'envisager cette lecture.

7 Un premier indice est fourni par le traitement réservé aux personnages qui pourraient sauver les chrétiens de la menace sarrasine. Parmi les noms propres regroupés dans l'index établi par Andrea Tarnowski dans son édition du texte, onze seulement appartiennent à des contemporains de Christine ; trois noms apparaissent deux fois et parmi eux, ceux de Tamerlan et de Châteaumorant. Les autres désignent des princes français et des personnages proches de ces derniers. Compte tenu des réserves à faire sur la fiabilité des informations tirées de ce relevé ${ }^{15}$, la présence de ces deux noms souligne l'intérêt de Christine pour l'actualité de l'Orient et, plus précisément, pour ceux qui s'opposent à l'avancée musulmane. Tamerlan, au début du livre, est présenté comme un musulman méritant la mort autant que le sultan turc. Dans le second passage le concernant, on le considère capable de réduire à néant le royaume du sultan d'Égypte qui est décrit quelques vers avant comme une menace pour les chrétiens. Le chef mongol semble donc avoir une action bénéfique pour ces derniers. Cette présentation favorable de Tamerlan s'appuie sur la connaissance des usages des peuples orientaux et sur une distinction des différentes forces présentes en Orient ${ }^{16}$ : Tamerlan est effectivement plus bienveillant vis-à-vis des chrétiens que les autres chefs musulmans ${ }^{17}$. Quant à Châteaumorand, il fournit un exemple de chevalier parfait dans le discours de Sagesse ${ }^{18}$, ce qui fait de lui un égal des chevaliers romains. Cette comparaison élogieuse et la longueur du passage qui lui est consacré peuvent être perçues comme un signe de soutien de la part de Christine. Que ce soit pour leur force ou pour leur vertu, ceux qui peuvent nuire aux «Sarrasins" possèdent une place privilégiée dans le Chemin de long estude. Ils sont clairement nommés. Si les bienfaits des actions du chef musulman ne sont que mentionnés, ils sont largement développés dans le cas de Châteaumorant. En insistant ainsi sur les deux chefs de guerre, Christine attire l'attention de Charles VI sur ceux qui éloignent la menace musulmane des frontières de la chrétienté.

8 De Constantinople au monastère de Sainte-Catherine, en s'arrêtant dans tous les lieux saints et en y accomplissant ses dévotions, Christine effectue un pèlerinage fictionnel, motif récurrent dans la littérature didactique de la fin du Moyen Âge ${ }^{19}$. L'écrivain s'y décrit comme un pèlerin qui avance sur les chemins de la vie et du savoir, y acquiert la sagesse, et donc le droit d'être écouté par le prince. Pourtant, contrairement à d'autres textes où le narrateur évolue dans un univers allégorique, Christine parcourt, en ce cas, des lieux géographiques connus. Certains de ces lieux sont empreints d'une forte 
charge émotive car ils appartiennent à l'histoire de la chrétienté. De ce fait, une des fonctions principales du récit de la visite du Proche-Orient est de rappeler la présence des lieux symboliques de la religion chrétienne en terre musulmane et de souligner de façon discrète la menace induite par cette situation. Ainsi le long passage sur Constantinople est construit de telle façon qu'il oppose la splendeur de la ville due à la sagesse et à l'habileté des Byzantins ${ }^{20}$ et les ruines contemporaines dont les "Sarrasins » sont considérés comme seuls responsables ${ }^{21}$. La destruction de l'œuvre des empereurs chrétiens et le fait que, parmi tous les monuments de Constantinople, seul un symbole de la religion chrétienne, l'église Sainte-Sophie, soit cité, suggèrent l'idée que les «Sarrasins » s'attaquent non seulement à une ville prestigieuse, mais de façon plus générale, à la civilisation occidentale et à sa richesse culturelle et religieuse. Lors de la visite de Jérusalem, Christine accède aux lieux saints sans obstacle, comme les pèlerins de cette époque. Pourtant l'idée d'une persécution apparaît clairement dans les vers à propos du sultan du Caire cités plus haut ${ }^{22}$. L'encouragement à défendre le royaume byzantin contre les Ottomans, à combattre les Égyptiens qui détiennent les lieux sacrés est donc présent de façon implicite dans l'ensemble du récit du voyage en Orient. La dévotion de Christine peut en effet être considérée comme un appel au roi «très-chrétien » à secourir les symboles de la chrétienté menacés par la présence sarrasine.

9 Malgré la lamentation sur les guerres qui déchirent l'humanité, la méditation sur les conflits du monde fournit également une allusion tacite à la croisade. En effet, Christine désire d'une part qu'aucun chrétien ne soit mêlé à la lutte entre Bayazid et Tamerlan et, d'autre part, elle souhaite voir celle-ci se terminer par la mort des deux adversaires. La violence de cette aversion est surprenante car elle encourage les maux que le discours semble condamner. De ce fait, le souhait de tenir les chrétiens à l'écart du conflit apparaît moins comme la volonté d'éviter une guerre que d'épargner la vie des soldats occidentaux.

De plus, les conflits qu'elle dénonce sont d'un type bien particulier puisqu'il s'agit uniquement de luttes fratricides, dictées par la convoitise :

Tous li mondes est empeschez

De guerre, et plus sont rent,

Tant mains aiment leur parenté

Et plus queurent sus l'un a l'autre

A armeures, lances sur fautre,

Ou ilz assaillent leurs voisins (vv. 340-345).

11 De même que les oiseaux, les animaux terrestres, les poissons s'entretuent, les «Sarrasins » se combattent entre eux, et pire encore, les chrétiens se font la guerre pour des conquêtes futiles. Le découpage de l'humanité entre "Sarrasins » et chrétiens dans les exemples qui suivent les vers cités donne l'impression qu'il s'agit de deux ensembles bien distincts, n'entretenant aucun lien de parenté. C'est pourquoi la dénonciation des conflits entre chrétiens et musulmans ne s'inscrit pas dans la déploration de Christine. En revanche, la critique de la guerre entre Anglais et Français, les tensions pour le pouvoir entre les oncles et le frère de Charles VI sont dénoncées de façon à peine sous-entendue. La déploration du Chemin de long estude doit être rapprochée de celle de Philippe de Mézières dans la Lettre à Richard II. Le sentiment de désolation face aux conflits qui déchirent les hommes s'efface rapidement pour laisser place à la dénonciation du danger engendré par l'effusion du sang chrétien : 
Qui pourroit descripre a plain les effusions du sanc humain entre les paiens, Sarrasins et chrestiens, qui a este espandu par les morteles guerres, qui ont este des le commencement du monde? Il seroit impossible. Mais toutesfois il seroit expedient a ceulz qui ont volente de commencier les guerres, et par especial encontre leurs freres crestiens, qu'il eussent en un miroir continuelment devant eulz l'effusion du sanc humain cy dessus recitee, pour refaindre leur perilleuse volente. Et ce souffice du sanc humain espendu paint et figure en la dicte sale descouverte, et tout au desplaisir de Dieu, et encontre la divine bonte, et a la destruction de la crestiente. ${ }^{23}$

L'idée d'une paix franco-anglaise autour de la croisade telle qu'elle est défendue par Philippe de Mézières dans l'ensemble de son œuvre et plus particulièrement dans la Lettre à Richard II $^{24}$ est sans doute moins séduisante après la défaite de Nicopolis. Pourtant, elle est peut-être, aux yeux de Christine de Pizan, préférable à la lutte fratricide que représente la guerre de Cent ans. La paix entre les royaumes occidentaux, fondement essentiel de la concorde au sein du corps politique dont la recherche guide l'ensemble de l'œuvre politique de Christine, semble n'être possible qu'au prix d'une autre guerre, une guerre plus « juste » orientée vers la sauvegarde de la chrétienté, et non vers l'accroissement des richesses personnelles.

13 La responsabilité du roi de France à l'égard de l'empereur byzantin ${ }^{25}$, venu dans les cours occidentales demander de l'aide quelque temps avant le début de la composition du texte, mais plus encore, la nécessité de combattre les musulmans qui, aux portes de l'Europe comme en Terre Sainte ${ }^{26}$, offensent la religion chrétienne et présentent un danger pour la civilisation occidentale, sont des idées politiques qui apparaissent en filigrane dans le Chemin de long estude, à l'arrière plan du voyage onirique et du débat sur le roi idéal. Elles sont logiquement concentrées dans les visites de Constantinople et d'Égypte, points culminants annoncés ou rappelés par d'autres éléments disséminés dans l'ensemble du texte, comme un système d'échos visant à remémorer au souverain, en cours de lecture, l'actualité orientale et la nécessité de défendre la chrétienté.

Contrairement au Chemin de long estude, le chapitre du Charles $V$ concernant le visiteur égyptien ne comporte aucun jugement négatif sur l'étranger. Tout souligne la générosité du sultan et l'amitié qui est à l'origine de sa démarche. Comme les autres anecdotes de la vie du roi, le récit de cette visite a pour unique fonction de souligner la sagesse de Charles V. La proposition du sultan de faire du roi français le gouverneur de son royaume met en évidence la renommée du souverain défunt ${ }^{27}$. De même, le refus de cette offre met en relief le désintéressement du roi, qui le pousse à se détourner des richesses promises par le sultan pour continuer à gouverner la France. Il privilégie l'intérêt de son pays face à son intérêt personnel. L'envoyé mamelouk et, à travers lui, le sultan restent des faire-valoir pour Charles V. Ainsi, les présents du roi de France bénéficient d'une description bien plus détaillée que ceux du sultan. Tout rappel d'une hostilité entre chrétiens et musulmans dans ce chapitre ferait de Charles $\mathrm{V}$ un allié des ennemis de la foi chrétienne en raison de l'accueil cordial qu'il réserve à l'ambassadeur et nuirait à la glorification du roi exemplaire.

Christine de Pizan utilise donc les deux aspects de l'Islam, civilisation à la fois inquiétante et fascinante, développés dans la littérature médiévale ${ }^{28}$, selon la leçon politique qu'elle veut donner. Pourtant, le Charles $V$ n'est pas dénué de toute référence à la croisade. Lors du récit de la visite de l'empereur Charles IV à la cour du roi de France, Christine décrit le spectacle donné pendant le dîner du jour de l'Épiphanie ${ }^{29}$. Ce spectacle raconte la prise de Jérusalem par Godefroy de Bouillon. Dans son article sur la 
visite de l'empereur dans le Charles $V$ et les Grandes Chroniques de France ${ }^{30}$, Françoise Autrand s'étonne de la rapidité avec laquelle Christine traite un événement qui est pourtant, selon l'historienne, le fait marquant de la soirée. Il faut ajouter que l'auteur ne s'attarde pas à narrer en détail les scènes représentées mais elle insiste sur la qualité technique des décors :

Estoit la cité grande et belle, de bois, peinte, à panonceaux et armes des Sarrazins, moult bien faitte, qui fu menée devant le dois, et puis la nef où Goudeffroy de Billon estoit $^{31}$

16 Cet intérêt pour l'aspect matériel de la représentation s'explique, au regard de l'ensemble du chapitre, par le souci de souligner la richesse déployée lors de ce dîner : les nappes sont dorées, la vaisselle en or et en argent, les mets rares et nombreux. La beauté du décor théâtral participe de l'abondance de biens précieux et ainsi, contribue à montrer la puissance de Charles V. Néanmoins, cet aspect du récit ne doit pas occulter le soutien de Christine pour les idées exprimées dans le spectacle. Le choix de consacrer quelques lignes à la représentation prouve l'intérêt de l'écrivain pour cet événement. Il faut sans doute voir dans ce choix narratif une fidélité au texte des Grandes Chroniques qui sert de source à l'œuvre de Christine. Toutefois, comme le remarque Françoise Autrand $^{32}$, l'auteur se détache souvent du récit du chroniqueur pour créer un texte personnel. C'est le principe du travail de compilation qui constitue également une des fonctions essentielles du métier d'écrivain tel que Christine le définit, notamment dans le Charles $V^{33}$. Au-delà de l'importance de ce spectacle signalée par les Grandes Chroniques, le choix narratif de Christine est porteur de sens et révèle le soutien de l'auteur pour le message d'appel à la croisade délivré par le spectacle. L'adhésion de Christine au désir de reconquête des lieux saints s'exprime également par les avis qu'elle émet sur l'enseignement donné lors de la représentation. Dans le récit pourtant concis de l'événement, elle exprime par deux fois son approbation de ce qui est montré : «laquelle hystoire ramentevoir estoit pertinent pour exemples donner à telx princes ", « et puis l'assault commencié et la cité prise, qui fu bonne chose à veoir ». En intégrant ces propos dans sa compilation, l'écrivain se les approprie. Le soutien de Christine à la croisade est manifeste, mais aussi son appui à la tentative des princes occidentaux de bâtir une alliance pour la reconquête de Jérusalem, que suppose le fait de donner un tel spectacle lors de la visite diplomatique d'un souverain étranger. Nous avons vu que cette idée est particulièrement présente dans l'œuvre de Philippe de Mézières. Il faut ajouter que ce dernier attribue également à l'empereur d'Allemagne une part de responsabilité dans la possession de Jérusalem par les musulmans ${ }^{34} . \mathrm{Si}$, comme le suggère Françoise Autrand ${ }^{35}$, cet auteur est à l'origine de la mise en scène, l'influence du précepteur de Charles VI, déjà remarquée dans le Chemin de long estude, sur la conception christinienne de la croisade se confirme. Le Charles $V$ contient donc un encouragement à aller combattre les « Sarrasins » en Orient.

17 Bien que discret, l'intérêt de Christine de Pizan pour la reconquête des lieux emblématiques de la foi chrétienne est présent dès ses premières œuvres politiques. Mais l'idée de croisade s'exprime différemment selon la nature du texte dans lequel elle s'inscrit. Le Chemin de long estude, œuvre littéraire, énonce clairement l'hostilité de Christine envers les musulmans. Au contraire, dans la prose historique du Charles V, dominée par le désir de faire reconnaître la véracité des faits présentés, l'épisode le plus long concernant l'Orient est dénué de toute incitation à la guerre. L'expression de l'idée de croisade semble donc associée à l'œuvre dont l'appartenance au domaine littéraire et à la fiction est la plus marquée. Le court passage du Charles V où Christine 
soutient la guerre en Orient confirme cette distinction entre œuvre artistique et traité politique en prose : l'affirmation de la nécessité de reconquérir Jérusalem prend place dans la description d'un spectacle dont Christine ne vante pas la fidélité au fait historique mais la beauté de l'artifice. Le fait que le Ditié de Jehanne d'Arc, texte versifié de soixante et onze strophes, contient un encouragement très clair à combattre en Terre Sainte souligne également la corrélation entre texte poétique et appel à la croisade.

L'étude des emplois du mot « sarrasin » apporte des arguments qui vont dans le même sens. Ce terme est en effet fortement lié dans la tradition littéraire aux chansons de geste, à la guerre et à une image des musulmans où la haine de l'infidèle, diable incarné, rivalise avec l'attirance pour le raffinement de l'art, de l'artisanat et de la philosophie ${ }^{36}$. Christine utilise à de nombreuses reprises le terme de "sarrasin » dans le Chemin de long estude. Il apparaît dans le récit du Charles V de l'« entremez » offert à l'empereur Charles IV. Dans l'épisode de l'ambassadeur,elle privilégie la fonction et l'origine géographique du visiteur étranger en employant les expressions " chevalier » et « soubdan » suivi ou non de «Babiloine ». On peut déduire de ces remarques lexicales que le premier texte, dont la fonction esthétique est plus affirmée, fait davantage appel au motif littéraire des «sarrasins " et à l'exotisme à la fois attirant et menaçant qui l'accompagne. Le second texte préfère la précision historique à un terme chargé d'imaginaire.

19 Si cette analyse se vérifie en partie, un examen plus précis en révèle les limites. Le mot "sarrasin » désigne une fois l'ambassadeur égyptien qui se présente à la cour de Charles V alors qu'il n'apparaît pas dans le récit de la visite du Proche-Orient du Chemin de long estude, sans que cela remette en cause le caractère topique du passage. Le terme "souldan » utilisé dans ce dernier texte est tout aussi empreint d'un exotisme hérité des œuvres littéraires des siècles précédents. Dans le Charles $V$, le témoignage de Christine, qui a assisté dans son enfance à la visite du Mamelouk, associé à l'écriture en prose, accrédite le récit et ôte partiellement le caractère topique des mots "sarrasins " et "souldan" pour les intégrer au vocabulaire historique. Christine de Pizan n'est d'ailleurs pas la seule à employer le premier de ces mots dans une œuvre historique, le Religieux de Saint-Denis l'utilise également ${ }^{37}$. Toutefois, la notion d'exotisme qui s'attache à ces termes ne dessert pas la volonté de Christine de souligner la grande renommée du roi Charles : il est reconnu comme un grand roi par un seigneur lointain, presque mythique. Cela contribue à faire du roi français un souverain lui-même légendaire. La fiction s'introduit donc dans la prose historique sans pour autant nuire à la construction du roi idéal, bien au contraire. Dans le Charles $V$, l'exactitude historique, pourtant revendiquée pour certifier le caractère sérieux du texte, est en fait secondaire par rapport au souci de l'auteur de composer un miroir du prince. Qu'importe donc si, par endroits, la littérature prime sur l'histoire, tant qu'elle sert la leçon politique.

L'idée de croisade est donc présente dans l'œuvre de Christine de Pizan dès les premiers textes politiques. Si ce motif reste rare dans l'ensemble de son œuvre, Christine l'utilise malgré tout comme un élément constitutif de la leçon politique qu'elle donne aux princes. Dans le Chemin de long estude, le contexte historique, notamment la menace turque toujours présente malgré la défaite de Bayazid ${ }^{38}$, la faiblesse de l'empereur de Constantinople, la possession des lieux importants de la religion chrétienne par les Mamelouks, donnent l'occasion à l'écrivain de rappeler au souverain son devoir de protéger la chrétienté contre ses ennemis au lieu de combattre 
les Anglais pour des questions d'intérêt personnel. Ce même devoir est évoqué lors de la visite de l'empereur d'Allemagne et ainsi rappelé à la mémoire des princes destinataires du Charles $V$.

21 La croisade, dans l'œuvre de Christine de Pizan, appartient à la fois à l'histoire, qu'elle soit passée ou contemporaine, et à la fiction. Associée au topos des "sarrasins ", elle sert à transformer des personnages historiques en modèles à suivre, en êtres de légende à imiter : Charles $\mathrm{V}$ reconnu par un sultan tellement lointain qu'il touche à la merveille devient lui aussi un être exceptionnel; dans le Ditié, Jeanne d'Arc doit conduire Charles VII en Terre Sainte ${ }^{39}$ pour vaincre les "sarrasins » et devient ainsi l'égale de Godefroy de Bouillon, un des neuf preux légendaires ${ }^{40}$. En idéalisant des personnages contemporains, proches des princes français, Christine leur offre des modèles auxquels il est aisé de s'identifier. Ils peuvent eux aussi devenir des souverains exemplaires, à condition de délaisser leurs intérêts privés et, en faisant preuve de courage et surtout de sagesse, sauver le royaume de la ruine auquel il est promis.

\section{NOTES}

1. Voir notamment la ballade "Pour conquerir de cuer la Saincte Terre", Euvres complètes d'Eustache Deschamps, éd. marquis de Queux de Saint-Hilaire et G. Raynaud, Paris, Société des Anciens Textes Français, 1878-1904, n 49, t. I, pp. 138-139.

2. Philippe de Mézières, Letter to King Richard II, éd. G.W. Coopland, Liverpool, Liverpool University Press, 1975 ; Epistre lamentable et consolatoire, éd. K. de Lettenhove, dans Chroniques de Froissart, t.16, Bruxelles, 1872 ; Le Songe du vieil pelerin, éd. G. W. Coopland, Liverpool, Liverpool University Press, 1969.

3. B. A. Caroll, « On the Causes of War and the Quest for Peace : Christine de Pizan and early peace theory ", dans Au champ des escriptures: III colloque international sur Christine de Pizan, dir. Eric Hicks, Paris, Champion, 2000, pp. 337-358.

4. Ditié de Jehanne d'Arc, éd. J. Kennedy et K. Varty, Oxford, 1977.

5. E. Jeffrey Richard, « French Cultural Nationalism and Christian Universalism in the Works of Christine de Pizan ", dans Politics, Genre and Gender: The Political Thought of Christine de Pizan, dir. M. Barban, Boulder, San Francisco, London, Westview Press, 1992, pp. 76-96.

6. «As Jeffrey Richards argues, in her earlier works, which are the more important in this context, Christine's nationalism was not jingoistic. ", op. cit., p. 352.

7. Le Chemin de longue étude, éd. et trad. A. Tarnowski, Paris, Librairie Générale Française, 2000, (Lettres gothiques). Nous abrégeons par la suite le titre sous la forme Chemin de long estude.

8. Le Livre des fais et bonnes meurs du sage roy Charles V, éd. S. Solente, Paris, Société de l'Histoire de France, 1936-1941 ; reprint Genève, Slatkine, 1977, 2 vol. Nous nous servons de l'abréviation Charles V.

9. C. Marchello-Nizia, "Entre l'histoire et la poétique le «Songe politique » ", Revue des Sciences humaines, 1981, $\mathrm{n}^{\circ} 183$, pp. 39-53.

10. «Ainsi plaist au tres redoubté susdit que le petit entendement de mon engin s'applique à ramener à memoire les vertus et fais du tres sereins prince, le sage roy Charles, ameur de sapience et toute vertu; des quelles choses, pour emplir le dit commandement, me suis informée, 
tant par croniques, comme par pluseurs gens notables encore vivans jadis ses serviteurs, de sa vie, condicions, meurs et ordre de vivre, et de ses fais particuliers », livre I, chapitre III, p. 9.

11. Vv 412-426.

12. Vv.1317-1318 et vv. 1326-1330

13. «Le jour que j'os cel oprobe/Fu le .v.e d'octobre/Cest an mille .cccc. » (vv. 185-187).

14. F. Autrand, Charles VI : La folie du roi, Paris, Fayard, 1986, pp. 257-260 et 342-343.

15. En effet, le nom de Charles VI n'apparaît qu'une fois alors que bien des termes désignent le roi dans la dédicace.

16. Voir S. Sasaki, «Châteaumorant et Le Chemin de Christine de Pizan : à propos des « ruines » de Constantinople" dans Et c'est la fin pour quoy sommes ensemble: Hommage à Jean Dufournet, t.1, Paris, Champion, 1993, pp. 1261-1270 : Christine tire ses informations de Châteaumorand.

17. Le but des conquêtes de Tamerlan est, officiellement, la propagation de l'Islam plutôt que la constitution d'un empire. Pourtant, lors de ses victoires, le chef mongol se montre amical envers les Chrétiens, notamment en libérant, après la prise de Brousse, capitale de l'Empire ottoman, le 3 août 1402, les prisonniers de la bataille de Nicopolis détenus par Bayazid. Ce geste le montre sous un jour favorable dans les cours occidentales à l'époque de l'écriture du Chemin de long estude. D'ailleurs, Charles VI et Henri IV ont envoyé des lettres de félicitations à Tamerlan lors de sa victoire. La libération des prisonniers occidentaux n'est sans doute pas étrangère à cette reconnaissance diplomatique. Voir le livre de J.-P. Roux, Tamerlan, Paris, Fayard, 1991 et A. Demurger, «Le Religieux de Saint-Denis et la croisade ", dans Saint-Denis et la royauté: Etudes offertes à Bernard Guenée, dir. F. Autrand, C. Gauvard et J.-M. Moeglin, Paris, Publications de la Sorbonne, 1999, pp. 181-196. L'attitude de Christine envers Tamerlan correspond certainement aux sentiments de la cour à son égard : une crainte face à sa puissance dévastatrice que nul ne semble arrêter mêlée à une gratitude pour son combat contre Bayazid et son ménagement des Chrétiens. Il semble moins dangereux que le sultan turc.

18. Vv. 4503-4570.

19. Voir notamment Le Songe du vieil pelerin de Philippe de Mézières et les premières lignes du Livre de l'Advision Cristine de Christine de Pizan, éd. C. Reno et L. Dulac, Paris, Champion, 2001, (Études christiniennes).

20. «Si louoie les ancians/Qui avoient tieulx essiens/Que faire firent tieulx ouvrages » (vv. 1217-1219).

21. Voir B. Ribémont, «Christine de Pizan : entre espace scientifique et espace imaginé (Le Livre du Chemin de long estude)", dans Une femme de lettres au Moyen Âge. Études autour de Christine de Pizan, dir.L. Dulac et B. Ribémont, p. 252.

22. Vv. 1317-1318.

23. Op. cit., p. 131.

24. Ibid., p. 123 : « Il est expedient que pour empetrer la vraie paix de la crestiente, pour l'union de nostre mere sainte eglise, et pour delivrer la terre sainte de la main de faulz prophete Mahommet, que nostre escharboucle figuree, la pierre d'aymant et nostre figure dyamant soye melle et trempe sagement et discretement ensemble en esperit, sans pompe, sans serimonies, et sanz gait, en grant humilite et amoureuse fraternite, ou precieux vin des vignes d'Engady, tant de foyz reciteez ». L'« escharboucle » et la « pierre d'aymant» désignent le roi d'Angleterre, le «dyamant» le roi de France.

25. Voir l'article de C. Cannon Willard, « Une source oubliée du voyage imaginaire de Christine de Pizan ", dans Et c'est la fin pour quoy sommes ensemble: Hommage à Jean Dufournet, t. I, Paris, Champion, 1993, p. 326 : «Que voulait-elle dire aux princes? Le contenu de ce message, « fait par manière poétique ", était-il que les princes avaient une responsabilité vis-à-vis de l'empereur et du monde qui dépassât les bornes de leur propre pays et leurs ambitions personnelles ? C'est surtout la date du poème qui soulève cette question pertinente. Il est sans doute évident que 
Boucicaut et Châteaumorant, parmi d'autres, le croyaient ; et il semble probable que Christine, en dédiant son poème au roi et aux princes français, se ralliait à une pareille idée » .

26. Comme le précise A. Demurger, (op. cit, pp. 195-196), Jérusalem n'est plus le but principal des croisades sous le règne de Charles VI. Cette ville est plutôt la destination de pèlerinages pacifiques, comme celui que Christine décrit dans son propre voyage. Pourtant, Jérusalem demeure toujours l'objectif d'un combat contre les infidèles qui passe d'abord par l'anéantissement des Turcs.

27. L. Dulac, «La figure de l'écrivain dans quelques traités en prose de Christine de Pizan », dans Figures de l'écrivain au Moyen Âge, dir. D. Buschinger, Göppingen, Kümmerle Verl., 1991, p. 118 : «L'ambassade du sultan d'Égypte, qui est également rappelée en termes personnels, doit sans doute ce traitement moins à l'étrangeté de l'événement qu'à sa valeur de symbole d'une splendeur monarchique disparue ".

28. Ph. Sénac, L'Image de l'autre: L'Occident médiéval face à l'Islam, Paris, Flammarion, 1983.

29. Christine parle de «.II. entremez » mais semble n'en décrire qu'un seul. Le texte des Grandes Chroniques de France est plus clair et précise que les deux spectacles, l'un présentant Godefroy de Bouillon sur sa nef et l'autre montrant la cité de Jérusalem aux mains des musulmans, se rejoignent pour n'en former qu'un seul: «Et lors se mistrent les deux entremés l'un contre l'autre et descendirent ceuls de la nef, et par belle et bonne ordenance vindrent donner assault à la dite cité et longuement l'assaillirent ", Chronique des règnes de Jean II et de Charles V: t. II 1364-1380, éd. R. Delachenal, Paris, Librairie Renouard, 1917, p. 242.

30. F. Autrand, «Mémoire et cérémonial: La Visite de l'empereur Charles IV à Paris en 1378 d'après les Grandes Chroniques de France et Christine de Pizan ", dans Une femme de lettres au Moyen Âge : Études autour de Christine de Pizan, op . cit., pp. 91-103. Voir plus particulièrement la page 97.

31. Op. cit., t. 2, p. 113.

32. Op. cit., p. 91 : «Christine de Pizan ne recopie pas le récit tel qu'il figure dans l'exemplaire des Grandes Chroniques qu'elle a sous les yeux. Elle en prend et elle en laisse, signalant ainsi ce qui a le plus de sens pour elle, pour son temps, pour le courant de pensée auquel elle appartient. »

33. Voir le chapitre XXI du livre II.

34. Op. cit., pp. 98-99: « il est assavoir, parlant moralment et grossement, quant a l'exposicion de la dicte parabole, que par le roy Malavise puet estre entendue la personne d'un roy crestien et catholique, representent en sa personne l'empereur de Romme et tous les roys crestiens catholiques. Et par la personne du roy Vigilant puet estre entendu proprement le souldain de Babiloine, comme il aparra cy dessus par les similitudes et concordances de l'efect des ii. Rois. [...] Et par deffaulte de bon gouvernement, c'est assavoir la sainte cite de Jherusalem, le premier fondement de la foy catholique, et, apres, tout le royaume, et toute la terre de promission, a este pardue, et acquise a grant victoire par le roy Vigilant, c'est assavoir par le souldain de Babiloine, qui en a eu la possession et c. ou ii ${ }^{\varsigma}$ ans, a grant honte et vitupere des roys crestiens, helas, malavises".

35. Op. cit., p. 97 : « On croit reconnaître dans cette mise en scène la main de Philippe de Mézières ou au moins son inspiration ».

36. Ph. Sénac, op. cit. p. 137.

37. Voir A. Demurger, op. cit., p. 184

38. Bayazid est vaincu par Tamerlan et fait prisonnier le 28 juillet 1402 à la bataille d'Ankara. Son fils Sulaïman réussit à fuir. Comme le précise J.-P. Roux (op. cit., p. 144) : «Les forces ottomanes n'étaient pas anéanties : elles étaient intactes en Europe et il en restait encore en Anatolie ", ce qui explique que Christine parle du danger turc au présent, sans mentionner le nom de Bayazid.

39. « Des Sarradins fera essart,/En conquerant la Sainte Terre. Là menra Charles, que Dieu gard ! Ains qu'il muire, fera tel erre./Cilz est cil qui la doit conquerre./Là doit-elle finer sa vie,/Et l'un et l'autre gloire acquerre./ là sera la chose assovye. », vv. 337-344. 
40. «Donc desur tous les preux passez,/ Ceste doit porter la couronne,/Car ses faiz ja monstrent assez/Que plus prouesse Dieu lui donne/Qu'à tous ceulz de qui l'on raisonne. », vv. 345-349. 